\title{
Erratum to: On the issue of defining the essence of the object of the intangible cultural heritage: historical retrospective and significance for the development of modern society
}

\author{
Muminat Doguzhaeva ${ }^{1, *}$, Nikolai Dorokhov ${ }^{1}$, Tatiana Kochemasova ${ }^{1}$, Natalia Nazarenko ${ }^{1}$, and Evgeniya Simaeva ${ }^{1}$ \\ ${ }^{1}$ Department of Legal Regulation of Economic Activity, Financial University under the Government of the Russian Federation, \\ 125993, 49 Leningradsky ave., Moscow, Russia
}

Original article: SHS Web of Conferences 55, 05009 (2018), https://doi.org/10.1051/shsconf/20185505009

There is a mistake in the surname of the third author, Tatiana Kochemasova, it should be:

Muminat Doguzhaeva ${ }^{1, *}$, Nikolai Dorokhov ${ }^{1}$, Tatiana Kochepasova ${ }^{1}$, Natalia Nazarenko $^{1}$, and Evgeniya Simaeva ${ }^{1}$ ${ }^{1}$ Department of Legal Regulation of Economic Activity, Financial University under the Government of the Russian Federation, 125993, 49 Leningradsky ave., Moscow, Russia 